\title{
Evaluación de la Dinámica de la Laguna de Apoyo mediante Trazadores Isotópicos y Geoquímicos
}

Heyddy Calderón Palmay Yelba Flores Meza

Centro para la Investigación en Recursos Acuáticos de Nicaragua, Universidad Nacional Autónoma de Nicaragua, Apdo. Postal 4598, Managua, Nicaragua. Correo electrónico: heyddy.calderon@cira-unan.edu.ni, yelba.flores@cira-unan.edu.ni

\section{Resumen}

$\mathrm{E}$ 1 objetivo de este estudio fue evaluar la dinámica de la Laguna de Apoyo usando herramientas isotópicas y geoquímicas. Esta laguna es de origen cratérico de $6.6 \mathrm{~km}$ de diámetro y $175 \mathrm{~m}$ de profundidad. La cuenca es endorreica, pequeña $\left(38 \mathrm{~km}^{2}\right)$, con un promedio de precipitación acumulada anual de $1454 \mathrm{~mm}$. Los datos limnimétricos indican una disminución del nivel de la laguna de $10 \mathrm{~m}$ en los últimos 30 años.

Se monitorearon parámetros fisicoquímicos y la composición isotópica $\left({ }^{18} \mathrm{O}\right.$ y $\left.\mathrm{D}\right)$ de las aguas de la laguna, manantiales, pozos y de la precipitación durante 2006 y 2007. A pesar de la presencia de una barrera poco permeable al Oeste de la Laguna de Apoyo, los resultados hidroquímicos indican aportes desde el Noroeste y Suroeste. Sin embargo, aún no se descarta recarga local. Los datos isotópicos muestran que la evaporación es muy significativa en el balance hídrico de la laguna. Se identificó una zona de descarga de la laguna al Noreste, hacia el acuífero aguas abajo. Debido a la influencia de termalismo es necesario extender el monitoreo para definir más en detalle la zona de descarga y finalizar el balance isotópico. Esto es muy importante dado el alto contenido de sales y arsénico en la laguna y la influencia que ésta puede tener en el acuífero de Granada, ubicado aguas abajo.

Palabras claves: laguna cratérica, geoquímica, balance isotópico
Recepcionado: 25 Noviembre 2009/ Aceptado: 09 Mayo 2010

\section{Abstract}

The objective of this study was to assess flow dynamics of the Apoyo Crater lake using isotopic and geochemical tools. This crater lake of $6.6 \mathrm{~km}$ of diameter is $175 \mathrm{~m}$ deep. The lake watershed is small, with an area of about $38 \mathrm{~km}^{2}$, annual precipitation is about $1454 \mathrm{~mm}$. In the last 30 years, the lake has suffered a $30 \mathrm{~m}$ decline in water level.

Physical-chemical parameters and isotopic composition $\left({ }^{18} \mathrm{O}\right.$ y $\left.D\right)$ of the lake, springs and wells were monitored during 2006 and 2007. Despite the presence of a low permeability barrier west of the lake, geochemical results indicate groundwater contribution from NW and SW directions. Nevertheless, local recharge has not been ruled out yet. 
Isotopic data show that evaporation is highly significant for the water balance of the lake. A discharge area was identified to the NE towards an important aquifer in the city of Granada. It is necessary to extend the monitoring network to better define the discharge area and close the water balance. This is extremely important since the lake water has a high salt and arsenic content, which may be altering the water quality of the Granada Aquifer.

\section{Introducción}

Nicaragua posee numerosas lagunas cratéricas pero son pocos los estudios hidroquímicos e hidrogeológicos que se han realizado sobre ellas. La laguna de Apoyo está ubicada sobre la franja volcánica del Pacífico de Nicaragua, entre los volcanes Masaya y Mombacho (Figura 1). Esta zona presenta numerosas fallas y gran actividad sísmica. Apoyo es una de las lagunas cratéricas más grandes de Nicaragua, con $6.6 \mathrm{~km}$ de diámetro, $175 \mathrm{~m}$ de profundidad $\mathrm{y}$ un área de $21 \mathrm{~km}^{2}$. Sin embargo, la cuenca superficial es pequeña con tan sólo $38 \mathrm{~km}^{2}$ y además endorreica. Estas características contribuyen poco a la recarga superficial, la cual es predominantemente precipitación directa y escorrentía. Aunque la laguna se localiza en el extenso y productivo acuífero Las Sierras, recibe escasa contribución de agua subterránea, al presentar una barrera hidrogeológica (Krasny, 1995). Esto se debe a los depósitos de lavas en la zona suroeste que impide la entrada del flujo subterráneo que drena desde el noreste y descarga al lago de Nicaragua. Además la precipitación media anual en el área es de $1454 \mathrm{~mm}$ y está por debajo de la evaporación en tanque que es de $1736 \mathrm{~mm} /$ año. En estas condiciones el nivel medio de la laguna ha registrado un descenso de casi $10 \mathrm{~m}$ en un período de unos 30 años. Para el año 1982 se reportó el nivel de $78 \mathrm{~m}$ sobre el nivel del mar (Sussman, 1982) y en el año 2005 el nivel medio fue de $69 \mathrm{~m}$. Este estudio evalúa la dinámica del flujo y la química de las aguas de este cuerpo de agua sumando información a estudios previos (Sussman, 1982; Espinoza, 1999; CIRA, 2006; Vásquez et al., 2007).

En este estudio se presenta la relación de la hidrogeología con la geoquímica y resultados preliminares de los trazadores isotópicos ( $\mathrm{D} y{ }^{18} \mathrm{O}$ ). Posteriormente se sugiere colectar más información para finalizar el balance isotópico de la laguna y estudiar el posible impacto de la laguna en los acuíferos localizados aguas abajo de la misma.

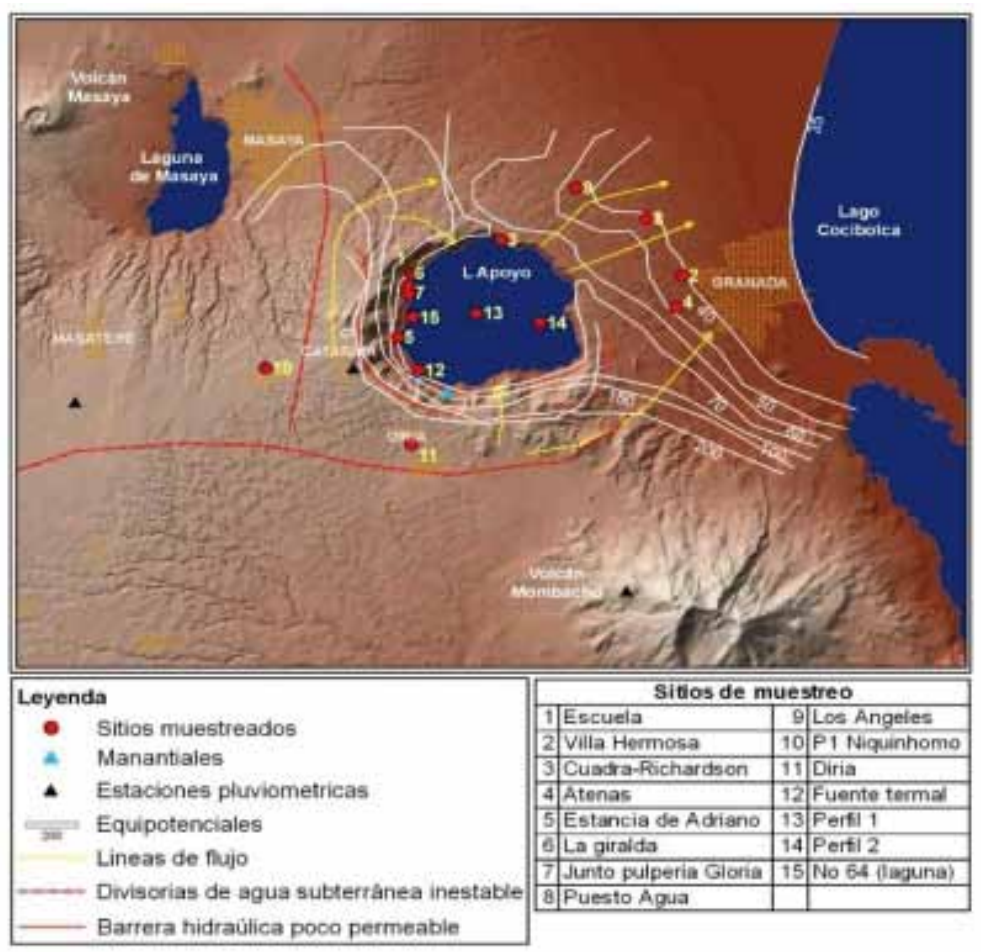

Figura 1. Dirección de flujo de agua subterránea y sitios de muestreo. Modificado de Krasny 1995. 


\section{Materiales y Métodos}

Se realizo el reconocimiento hidrológico de la zona de estudio, inventariando pozos y manantiales, midiendo niveles de agua subterránea y parámetros de campo ( $\mathrm{pH}$, conductividad y oxigeno disuelto) en aguas subterráneas y superficiales. Esta información fue organizada y analizada usando ArcGIS 9.2 y con ella se se hizo la selección de los sitios de de muestreo para análisis isotópicos, los cuales fueron realizados por espectrometría de masa en la Universidad de Waterloo, Canadá. Los análisis fisicoquímicos fueron realizados en el Centro para la Investigación de Recursos Acuáticos de Nicaragua (CIRA/UNAN) usando el Standard Method for the Examination of Water de la American Public Health Association (APHA, 1999).

El monitoreo fisicoquímico e isotópico para evaluar cambios temporales y espaciales en la hidroquímica y composición isotópica $\left({ }^{18} \mathrm{O}\right.$ y D) del agua de la laguna se basó en muestreos en época lluviosa (Sep. 2006) y en época seca (Abril 2007). Para evaluar los cambios horizontales, se colectaron muestras de agua en diferentes sitios de la laguna, complementado con dos perfiles verticales, para analizar cambios con la profundidad (Figura 1), tomando muestras desde la superficie hasta el fondo de la laguna $(175 \mathrm{~m})$. Los resultados de los análisis del agua de la laguna se correlacionaron con muestras de agua de siete pozos tanto en la posible zona de recarga, como en la de descarga. También se colectaron muestras de agua de dos manantiales y una fuente termal. Además, se monitoreó mensualmente, la composición isotópica de la precipitación en los alrededores de la laguna durante la época lluviosa y la seca para construir la línea de agua meteórica local. Se colectaron muestras en tres estaciones pluviométricas cercanas a la laguna y de una estación localizada a la orilla de la misma. Las muestras para el análisis de isótopos fueron procesadas en el laboratorio de la Universidad de Waterloo.

\section{Resultados y Discusión}

\section{Hidroquímica}

Las aguas de la Laguna de Apoyo se clasifican como $\mathrm{Cl}-\mathrm{Na}^{+}$y los valores medidos de conductividad llegan hasta $5958 \mu \mathrm{S} / \mathrm{cm}^{2}$. Datos históricos que datan desde 1993 (CIRA, 2006), muestran que las concentraciones de los iones principales y la conductividad han aumentado en el tiempo, lo cual puede relacionarse al descenso del nivel de la laguna. Los perfiles físico-químicos no muestran estratificación en la laguna ni variaciones horizontales significativas.

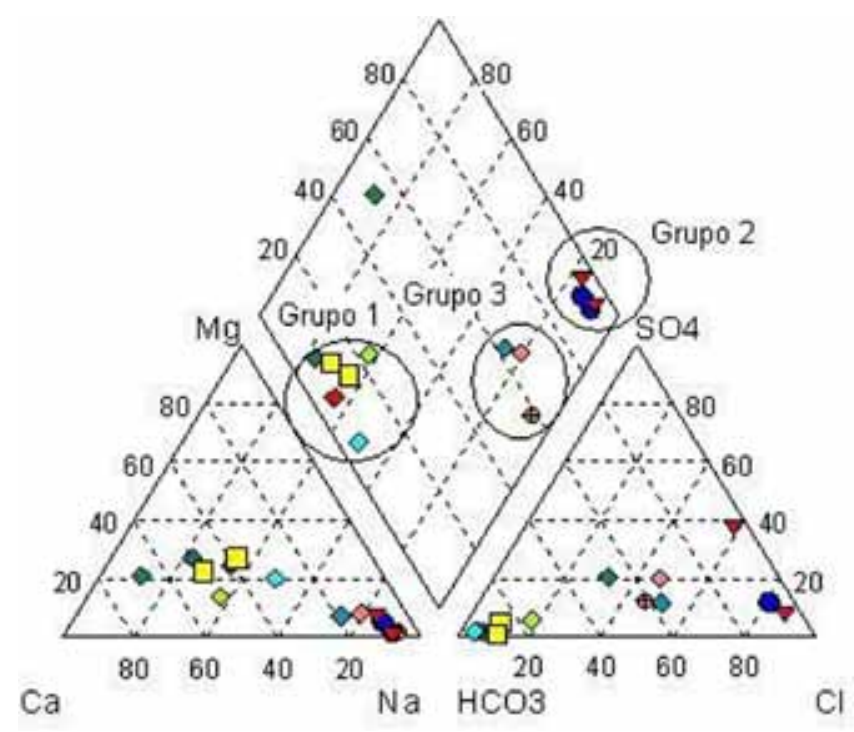

Figura 2. Resultados físico-químicos. PE: pozos excavados; PP: pozos perforados.

Los resultados se graficaron en un diagrama de Piper (Figura 2), donde se distinguen claramente tres grupos: 1) aguas meteóricas recientes de baja conductividad (350-480 $\mu \mathrm{S} / \mathrm{cm})$ y tipo $\mathrm{HCO}_{3}-\mathrm{Ca}-$ $\mathrm{Mg}$ y $\mathrm{HCO}_{3}-\mathrm{Ca}-\mathrm{Na}, \mathrm{HCO}_{3}-\mathrm{Na}-\mathrm{Ca}$. Estos tipos de agua han sido identificado como de recarga o poco recorrido para el acuífero Las Sierras (Hetch, 1995). A este grupo pertenecen los pozos perforados al SO y E de la laguna, alimentados por el acuífero las Sierras (Figura 2) y pozos excavados y manantiales 
dentro del cráter que sugieren entrada de agua subterránea a ella. 2) aguas $\mathrm{Cl}-\mathrm{Na}$ y $\mathrm{Cl}-\mathrm{Na}-\mathrm{SO}_{4}^{-2}$ de muy alta conductividad (3300 A $6320 \mu \mathrm{S} / \mathrm{cm}$ ). Estas corresponden a la laguna y fuentes termales y 3) aguas $\mathrm{HCO}_{3}-\mathrm{Cl}-\mathrm{Na}$ de alta conductividad ( $930 \mathrm{a}$ $2670 \mu \mathrm{S} / \mathrm{cm}$ ), probablemente producto de la mezcla del acuífero regional y aguas termales profundas.

\section{Arsénico}

La presencia de arsénico en las aguas subterráneas $(4.65$ a $54.40 \mu \mathrm{g} / \mathrm{l})$ está relacionada a la presencia de fallas y termalismo en la zona. Sin embargo, la laguna podría ser una fuente de arsénico para los pozos ubicados en la zona de descarga, como en el caso del sitio Puesto de Agua (Figura 1).

\section{Composición isotópica}

Los datos isotópicos de precipitación permitieron caracterizar la línea meteórica local de acuerdo a la ecuación $\delta \mathrm{D}=7.68 \delta^{18} \mathrm{O}+11.35$ (Figura 3) . $\mathrm{El}$ agua de la laguna muestra valores isotópicos altamente enriquecidos que son típicos del proceso de evaporación.

Las aguas subterráneas se dividen en dos grupos, uno más empobrecido en isótopos pesados y corresponde a las aguas del grupo hidroquímico 1 (HQ1), (Figura
3). E1 otro grupo tiene un contenido isotópico más enriquecido que muestra el efecto del proceso de evaporación. En este último se encuentran pozos del grupo hidroquímico 3 (HQ3), (Figura 3).

La baja permeabilidad de las rocas en la zona Oeste de la laguna provoca el desvío del flujo regional del acuífero Las Sierras hacia el Norte y NE hacia el Lago Cocibolca. A pesar de esa barrera poco permeable, según los resultados físico-químicos, la laguna parece recibir alimentación subterránea desde el NO y SO.

La salinidad, el tipo hidroquímico y la composición isotópica de las aguas en los pozos ubicados en estas zonas de la laguna, sugieren aguas recientes de poco recorrido que coinciden con las aguas subterráneas al Oeste de la laguna.

La presencia de manantiales sugiere también la existencia de pequeños acuíferos colgados en el interior del cráter, su química y contenido isotópico coinciden con la del flujo subterráneo que aparentemente alimenta la laguna, pero no se descarta el aporte de recarga local ocurrida en el interior del cráter por precipitación.

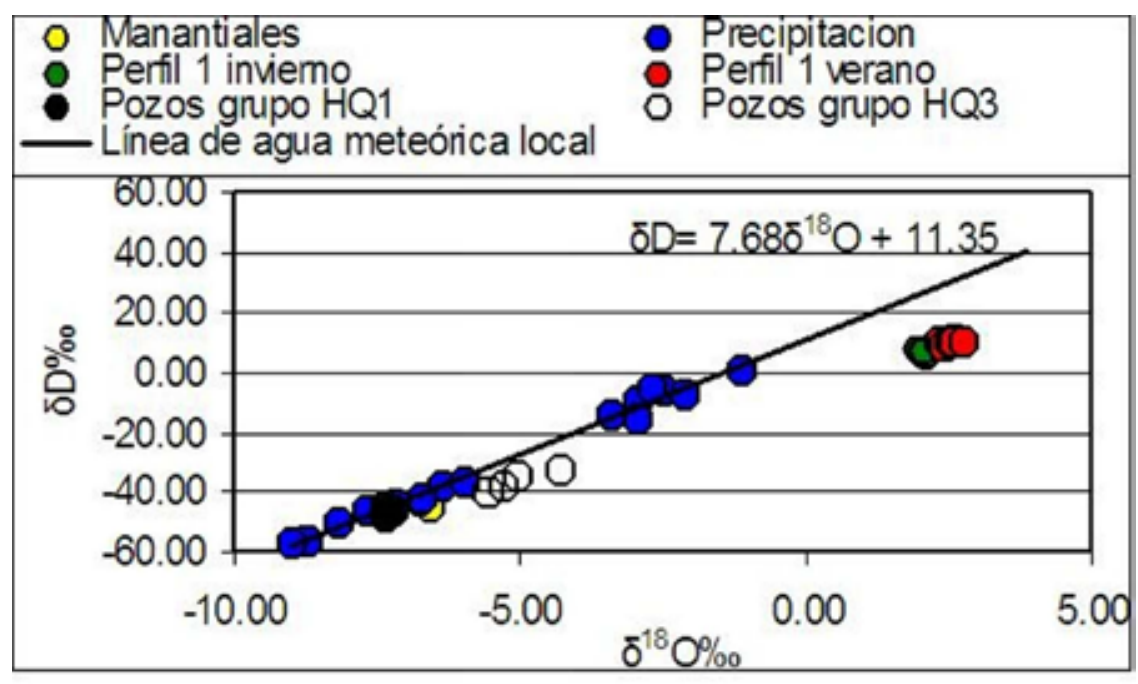

Figura 3. Resultados isotópicos de fuentes de agua en la zona de estudio 


\section{Conclusiones}

A partir del mapeo hidrogeológico y los datos químicos e isotópicos se ha determinado una zona de descarga de la laguna en el sector NE hacia el acuífero aguas abajo cerca de Granada, la cual tendría unos 3km de ancho. En esta zona se han identificado aguas con contenido isotópico más enriquecido que el flujo regional, indicando evaporación y sugiriendo una contribución de parte de la laguna. Sin embargo aún no se ha diferenciado completamente la zona de descarga debido a la influencia del termalismo que produce aguas con una composición química similar a la que descarga de la laguna.

Para completar el balance isotópico y determinar el volumen de agua con alto contenido de sales y arsénico que entra desde la laguna al acuífero aguas abajo es necesario extender la caracterización química e isotópica en la zona de descarga.

\section{Bibliografía}

American Public Health Association. (1999). Standard Methods for the Examination of Water and Wastewater. 20 th Ed. Washington.

CIRA/UNAN 2006. Informe sobre el Lago de Apoyo. Limnología, calidad de agua, hidrogeología e hidrogeoquímica. Reporte Interno, CIRA/UNAN.

Espinoza Ruiz M. (1999). Estudio hidrogeológico del acuífero de Granada, Nicaragua. Tesis de Maestría, Universidad de Costa Rica.

Krasny J. 1995. Mapa hidrogeológico de la zona del Pacifico de Nicaragua. INETER-GTZ.

Sussman D. 1982. The geology of Apoyo caldera. Master thesis. Dartmouth College. Hanover, New Hampshire.

Vázquez-Prada D, Ortega Fernández J, Alonso Marin E, Cerrato Mairena D. 2007. Estudio hidrogeológico y gestión de los recursos hídricos en la cuenca de la Laguna de Apoyo, Nicaragua. AMICTLAN-Geólogos del Mundo. 